\title{
Vibration-Based Damage Detection and Evaluation of Sheet Materials using a Remote Acoustic Excitation
}

\author{
Mfoumou, E. M., Hedberg, C. and Kao-Walter, S. \\ Blekinge Institute of Technology, School of Engineering \\ 37179 Karlskrona, Sweden *
}

\begin{abstract}
A simple method of damage severity assessment on sheet materials is suggested and proved by theory and experiment. The investigated defect types are in forms of added mass and crack. The method is based on the frequency shift measurement of a material vibrating as a membrane subjected to static tension and irradiated by acoustic wave. It is shown both theoretically and experimentally that the natural frequency of the damaged membrane is shifted relative to its position in the ideal material. A local increase in thickness (or addition of mass) shifts the natural frequency down, while a crack shifts the frequency up. The method can be considered as acoustic weighing through the frequency shift. The sensitivity of this method can be high because frequency measurement is one of the most accurate measurements in physics and metrology.
\end{abstract}

Keywords: Frequency shift, health monitoring, acoustic weighing.

\section{Introduction}

The interest in the ability to monitor sheet materials and detect damage at the earliest possible stage is universal throughout the civil, mechanical, and aerospace engineering communities, where safety, reliability and quality control have a large impact on such materials' application fields. A well-known example is the laminate used for liquid food packages, which is made of several layers of thin sheets including polymer film, aluminum foil and paperboard. A defect in such a material will alter the mechanical properties of the package, having a great influence on the behavior and the functionality during its lifetime.

For the purposes of this paper, paperboard (100 $\mu \mathrm{m}$ thick) is investigated for damage severity assessment. Damage is defined in the forms of local increase in the material's standard thickness, and local separation in the material (without lost of mass). Implicit in this definition of damage is that the concept of damage is not meaningful without a comparison between two different states of the material, one of which is assumed to represent the initial and undamaged state.

Most of the currently used damage identification methods belong to one of the following categories: visual or localized experimental methods such as acoustic or ultrasonic methods, magnetic field methods, radiography, eddy-current methods or thermal field methods [1]. All of these experimental techniques

\footnotetext{
*Contact: etienne.mfoumou@bth.se
} 
require that the vicinity of the damage is known a priori and that the portion of the material being inspected is readily accessible. The need for quantitative global damage detection methods has led to the development and continued research of methods that examine changes in the vibration characteristics of the structure. The usual approach of these methods consists in the determination of modal parameter changes due to the presence of the defect $[2,3]$.

This paper is therefore a contribution to the advances of vibration-based technique by providing a firm mathematical and physical foundation to the issue related to the detection of inhomogeneity in sheet materials. It also confirms the high sensitivity of the frequency measurement in the field of physical measurements. Experiments and simulations are conducted to support the study. In the experimental part, a low frequency non-contact excitation with a loudspeaker is performed, and the response of the material is recorded through a laser Doppler vibrometer, while simulation is performed in a finite element code.

\section{Vibration of membrane containing an added mass}

\subsection{Theory}

In accordance with the conditions of the experiment described in Figure 1, consider the sample of paper as a membrane subjected to tension.

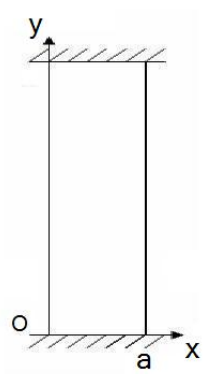

(i)

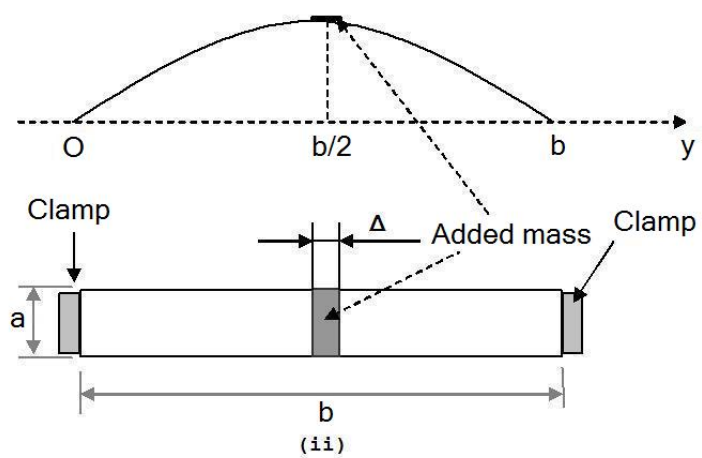

Figure 1: Specimen without (i) and with (ii) defect in form of added mass m'.

The equation of motion of the membrane, which lies in the plane of Cartesian coordinate system, has the form [4]:

$$
\frac{\partial^{2} \xi}{\partial t^{2}}-c^{2}\left(\frac{\partial^{2} \xi}{\partial x^{2}}+\frac{\partial^{2} \xi}{\partial y^{2}}\right)=\frac{p(x, y, t)}{\rho h}
$$

Here $\xi$ is the displacement of membrane along the $\mathrm{z}$ - axis from its equilibrium position $\mathrm{z}=0, c=\sqrt{T /(\rho h)}$ is the velocity of propagation of bending wave which is determined by the tensile force $T$ per unit length of boundary of membrane, $\rho$ is the density and $h$ is the thickness of the membrane. The external pressure $p(x, y, t)$ is a function of time and of spatial coordinates. If the pressure is zero, the free vibration of a rectangular membrane can be represented as a series of natural modes:

$$
\xi=\sum_{m, n=0}^{\infty} \xi_{m n}=\sum_{m, n=0}^{\infty} A_{m n} \cos \left(m \pi \frac{x}{a}\right) \sin \left(n \pi \frac{y}{b}\right) \sin \left(\Omega_{m n} t+\varphi_{m n}\right) .
$$


Here the constants $A_{m n}, \varphi_{m n}$ are amplitude and phase, $\Omega_{m n}$ is the natural frequency of mode,

$$
\Omega_{m n}=c \sqrt{\left(\frac{\pi m}{a}\right)^{2}+\left(\frac{\pi n}{b}\right)^{2}}, \quad m, n=0,1,2,3, \ldots
$$

and $a, b$ are the dimensions of the membrane. Each mode in the solution (2) satisfies the boundary conditions

$$
\xi_{m n}(x, y=0)=0, \quad \xi_{m n}(x, y=b)=0, \quad \frac{d \xi}{d x}(x=0, y)=0, \quad \frac{d \xi}{d x}(x=a, y)=0 .
$$

The conditions (4) correspond to the immovable boundaries at $y=0, y=b$ and to the free boundaries at $x=0, x=a$. If the membrane is loaded by an added mass $m^{\prime}$ located at $x=x_{0}, y=y_{0}$, the behaviour of that mass is governed by the equation of motion

$$
m^{\prime} \frac{d^{2} \xi}{d t^{2}}=-S p(x, y, t)
$$

where $S=l_{1} l_{2}$ is the area of contact between the mass and membrane. The equation (1) with account for (5) has the following form:

$$
\frac{\partial^{2} \xi}{\partial t^{2}}-c^{2}\left(\frac{\partial^{2} \xi}{\partial x^{2}}+\frac{\partial^{2} \xi}{\partial y^{2}}\right)=-\frac{m^{\prime}}{\rho h S} \frac{\partial^{2} \xi}{\partial t^{2}} \delta\left(\frac{x-x_{0}}{l_{1}}\right) \delta\left(\frac{y-y_{0}}{l_{2}}\right) .
$$

The Dirac $\delta$-functions are used here for simplicity, instead of continuous functions describing distribution of added mass on the surface of membrane. The $\delta$-function can be expanded in a Fourier series on the membrane modes:

$$
\begin{gathered}
\delta\left(\frac{x-x_{0}}{l_{1}}\right)=\frac{l_{1}}{a}+2 \frac{l_{1}}{a} \sum_{m=1}^{\infty} \cos \left(\pi m \frac{x_{0}}{a}\right) \cos \left(\pi m \frac{x}{a}\right), \\
\delta\left(\frac{y-y_{0}}{l_{2}}\right)=2 \frac{l_{2}}{b} \sum_{n=1}^{\infty} \sin \left(\pi n \frac{y_{0}}{b}\right) \sin \left(\pi n \frac{y}{b}\right) .
\end{gathered}
$$

It is necessary now to insert expansions $(7),(8)$ and (2) into equation (6). For the modes $\xi_{0 n}$ we get the dispersion law:

$$
-\omega_{0 n}^{2}+c^{2}\left(\frac{\pi n}{b}\right)^{2}=\frac{2 m^{\prime}}{M} \omega_{0 n}^{2} \sin ^{2}\left(\pi n \frac{y_{0}}{b}\right) .
$$

Here $M=\rho h a b$ is the mass of membrane. Because $\Omega_{0} n=\pi n c / b$ is the natural frequency of the unloaded membrane $\left(m^{\prime}=0\right)$, the frequency of the loaded one will be different:

$$
\omega_{0 n}=\frac{\Omega_{0 n}}{\sqrt{1+\frac{2 m^{\prime}}{M} \sin ^{2}\left(\pi n \frac{y_{0}}{b}\right)}}
$$

and for $m^{\prime}<<M$ we have

$$
\omega_{0 n} \approx \Omega_{0 n}\left[1-\frac{m^{\prime}}{M} \sin ^{2}\left(\pi n \frac{y_{0}}{b}\right)\right]
$$

After the $\approx$ sign the simplified expression is written which is valid for loading mass small compared with the mass of membrane $\left(m^{\prime}<<M\right)$. It follows from formula (11), that the frequency decreases with increase in loading. It depends also on the position of mass. If it is located in the maximum, the sine 
in (11) equals to unity and the frequency shift has its maximum. In the opposite case, if the mass $m^{\prime}$ is located in the node, the sine is zero and the added mass does not influence the frequency of vibration.

For the general modes $\xi_{m n}, m \neq 0$, a different dispersion law is valid:

$$
-\omega_{m n}^{2}+c^{2}\left[\left(\frac{\pi m}{a}\right)^{2}+\left[\left(\frac{\pi n}{b}\right)^{2}\right]=\frac{4 m^{\prime}}{M} \omega_{m n}^{2} \cos ^{2}\left(\pi m \frac{x_{0}}{a}\right) \sin ^{2}\left(\pi n \frac{y_{0}}{b}\right)\right.
$$

Consequently, the analog to formula (10) is

$$
\omega_{m n}=\frac{\Omega_{m n}}{\sqrt{1+\frac{4 m^{\prime}}{M} \cos ^{2}\left(\pi m \frac{x_{0}}{a}\right) \sin ^{2}\left(\pi n \frac{y_{0}}{b}\right)}}
$$

and for $m^{\prime}<<M$ we have

$$
\omega_{m n} \approx \Omega_{m n}\left[1-\frac{2 m^{\prime}}{M} \cos ^{2}\left(\pi m \frac{x_{0}}{a}\right) \sin ^{2}\left(\pi n \frac{y_{0}}{b}\right)\right]
$$

The theory developed above offers an idea on how one can detect an inhomogeneity in form of added mass using a measurement of the frequency shift. In other words, we use "acoustic weighing" of the specimen. The frequency measurement is known to be one of most accurate physical measurement $[5,6]$. Consequently, one can expect that the sensitivity of this method will be acceptable for nondestructive testing of sheet materials.

The sample studied is a rectangular strip of paper as shown in Figure 1. The addition of mass is a short strip of the same material, with the same width as the membrane. The strip is attached at the center of the membrane $(y=b / 2)$. The mode $\xi_{01}$ is excited by a loudspeaker. Consequently, the frequency shift is determined by formula (10):

$$
\frac{\Omega_{01}-\omega_{01}}{\Omega_{01}}=1-\frac{1}{\sqrt{1+\frac{2 m^{\prime}}{M}}}
$$

For $m^{\prime}<<M$ we have

$$
\frac{\Omega_{01}-\omega_{01}}{\Omega_{01}} \approx \frac{m^{\prime}}{M}
$$

For the mass of the specimen being small in comparison with the mass of membrane, the relative shift equals to the ratio of these masses.

\subsection{Experiment}

The case study investigated in this work is a single layer material (paperboard) clamped at the upper and lower edges in a tensile test machine, making the edges immovable (nodes). As a membrane has no compression or bending stiffness, it must be pre-stressed in order to act as a structural element. Therefore, the specimen is slightly loaded (within its elastic region) just enough to produce bending waves from a periodical acoustic load.

The specimen is preloaded at $17 \mathrm{~N}$ and has a mean thickness of $h=0.1 \mathrm{~mm}$. The length is $b=650$ $\mathrm{mm}$ and the width is $a=12 \mathrm{~mm}$. The acoustic load is provided by a loudspeaker having $4-8 \mathrm{Ohm}$ 
impedance, and a broad frequency range of 70 to $20.000 \mathrm{~Hz}$, centered at the middle of the specimen. Using an Agilent function generator the excitation frequency can be tuned continuously. A picture of the experimental setup is shown in Figure 2.

The specimens are excited using a $10 \mathrm{~V}$ sine sweep signal between 0 and $900 \mathrm{~Hz}$, which is sent to the loudspeaker through a function generator. The middle of the specimen is chosen as region of excitation, leading to strong excitation of odd modes of vibration. The method is to monitor the amplitude of the signal received in the time domain by a VS-100 OMETRON laser vibrometer, using A scans at the middle of the material. The laser signal fed to a high-pass/low-pass filter assures that only the excited frequency range is included in the velocity reading.

Small strips of mass $12 \mathrm{mg}$ (2.25\% of the total mass of the specimen) are successively added to the middle of the specimen length. The fundamental frequency shift is monitored for 1-10 added strips.

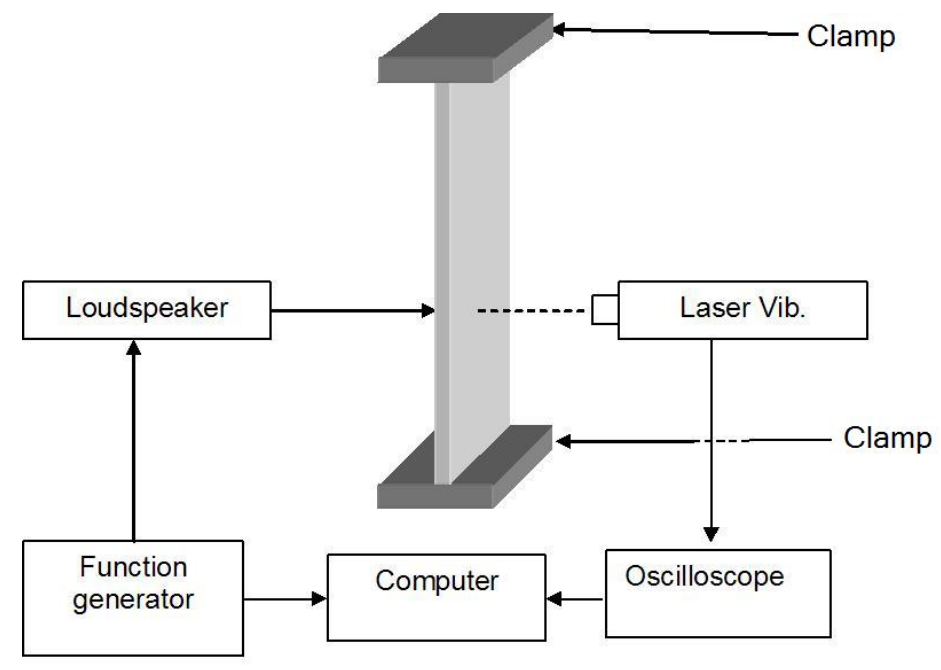

Figure 2: Experimental configuration.

\section{$2.3 \quad$ Results}

By inducing a defect in form of added mass the dominant fundamental eigenfrequency shifts to the left because the frequency is inversely proportional to the square root of the mass, as shown in equation (10). Table 1 shows the calculated fundamental frequency corresponding to the undamaged specimen, and illustrates the experimentally observed frequency shifts of the fundamental mode with addition of a mass corresponding to $2.25 \%$ of the total mass of the membrane.

\begin{tabular}{|l|c|c|}
\hline & Theory & Experiment \\
\hline Intact & 110.7 & 108.21 \\
\hline Damaged & 108 & 105 \\
\hline
\end{tabular}

Table 1: Fundamental frequencies for intact and damaged specimens.

A good agreement is obtained between the analytical (equation (15)) and experimental results, for a defect located at the middle of the specimen. This can be seen for the first mode resonance peak shift in Figure 3. 


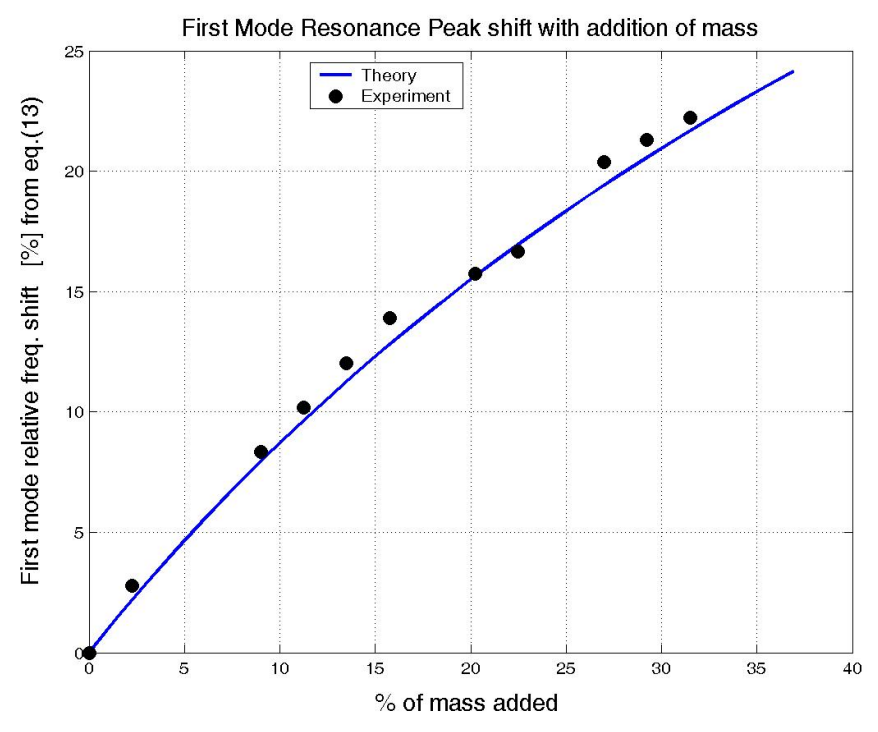

Figure 3: First mode relative frequency shift with addition of mass.

The variation tends to be somewhat nonlinear with increasing mass, showing that some nonlinear dependence has to be taken into account.

\section{Vibration of membrane containing a mass deficiency (crack)}

\subsection{Theory}

Let the membrane contain a crack as shown in Figure 4. Such a defect can be considered as a local opening in the material without reduction of the global mass of the structure.

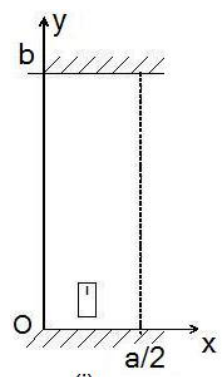

(i)

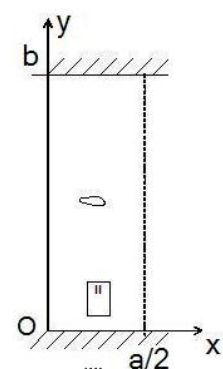

(ii)

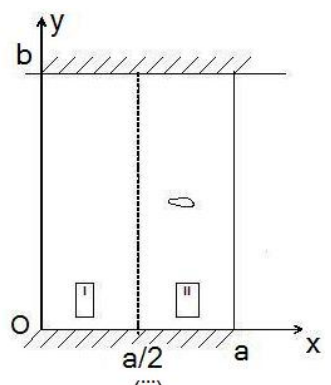

(iii)

Figure 4: Specimen configuration for hand-made small defect.

The membrane consists of two sections of equal width but with different velocities of bending waves. If these sections are isolated and vibrate independently their motions are governed by two different equations like (1):

$$
\frac{\partial^{2} \xi^{(1,2)}}{\partial t^{2}}-c_{1,2}^{2}\left(\frac{\partial^{2} \xi^{(1,2)}}{\partial x^{2}}+\frac{\partial^{2} \xi^{(1,2)}}{\partial y^{2}}\right)=0
$$

Index 1 here corresponds to the left (figure 4-(i)), and 2 - to the right-hand (figure 4-(ii)) section. Let 
the membranes vibrate in the $\xi_{m 1}$ mode. The solutions to (17) are

$$
\xi^{(1,2)}=A^{(1,2)}(x) \sin \left(\pi \frac{y}{b}\right) \cos \left(\omega^{(1,2)} t\right)
$$

If $A^{(1,2)}(x)=\cos (m \pi x / a)$, the natural frequencies are equal to

$$
\omega_{m 1}^{(1,2)}=\sqrt{\left(\frac{\pi}{b} c_{1,2}\right)^{2}+\left(m \frac{\pi}{a} c_{1,2}\right)^{2}}
$$

Substituting (18) into (17) we derive:

$$
\frac{d^{2} A^{(1,2)}}{d x^{2}}+\left(\frac{\omega^{(1,2) 2}}{c_{1,2}^{2}}-\frac{\pi^{2}}{b^{2}}\right) A^{(1,2)}=0
$$

In case $A^{(1,2)}(x)=$ const, we have the fundamental mode for which $\omega^{(1,2)}=(\pi / b) c_{1,2}$. Let $c_{2}<c_{1}$ and, consequently, $\omega_{2}<\omega_{1}$.

If the two sections of the membrane are joined (figure 4-(iii)), they vibrate with the frequency $\omega$, which lies between $\omega_{2}$ and $\omega_{1}$ :

$$
\omega_{2}<\omega<\omega_{1}
$$

We now seek the solutions of equations (17) in the form:

$$
\xi^{(1,2)}=A^{(1,2)}(x) \sin \left(\pi \frac{y}{b}\right) \cos (\omega t)
$$

where $\omega$ is an unknown frequency. Substituting (22) into (17), we obtain:

$$
\begin{aligned}
& \frac{d^{2} A^{(1)}}{d x^{2}}+\left(\frac{\omega^{2}}{c_{1}^{2}}-\frac{\pi^{2}}{b^{2}}\right) A^{(1)}=0 \\
& \frac{d^{2} A^{(2)}}{d x^{2}}+\left(\frac{\omega^{2}}{c_{2}^{2}}-\frac{\pi^{2}}{b^{2}}\right) A^{(2)}=0
\end{aligned}
$$

The coefficients in front of $A^{(1,2)}$ have different signs. In fact:

$$
\frac{\omega^{2}}{c_{1}^{2}}-\frac{\pi^{2}}{b^{2}}=\frac{\omega^{2}}{c_{1}^{2}}-\frac{\omega_{1}^{2}}{c_{1}^{2}}<0, \quad \frac{\omega^{2}}{c_{2}^{2}}-\frac{\pi^{2}}{b^{2}}=\frac{\omega^{2}}{c_{2}^{2}}-\frac{\omega_{2}^{2}}{c_{2}^{2}}>0
$$

The inequalities (25) are valid because the ratio (21) between the frequencies exists. Consequently, equations (23) and (24) can be rewritten as follow:

$$
\frac{d^{2} A^{(1)}}{d x^{2}}-k_{1}^{2} A^{(1)}=0, \quad \frac{d^{2} A^{(2)}}{d x^{2}}+k_{2}^{2} A^{(2)}=0
$$

with

$$
\kappa_{1}^{2}=\frac{\pi^{2}}{b^{2}}-\frac{\omega^{2}}{c_{1}^{2}}, \quad \kappa_{2}^{2}=\frac{\omega^{2}}{c_{2}^{2}}-\frac{\pi^{2}}{b^{2}}
$$


The well-known solutions to equations (24) are

$$
\begin{gathered}
A^{(1)}=\alpha_{1} \cosh \left(\kappa_{1} x\right)+\beta_{1} \sinh \left(\kappa_{1} x\right) \\
A^{(2)}=\alpha_{2} \cos \left(\kappa_{2} x\right)+\beta_{2} \sin \left(\kappa_{2} x\right)
\end{gathered}
$$

$\alpha_{1}, \alpha_{2}, \beta_{1}$ and $\beta_{2}$ are constants.

Solving $\left.\frac{d A^{(1)}}{d x}\right|_{x=0}=0$ and $\left.\frac{d A^{(2)}}{d x}\right|_{x=a}=0$ for $\beta_{1}$ and $\beta_{2}$ and substituting into (26) and (27) leads to the simpler forms:

$$
\begin{gathered}
A^{(1)}=\alpha_{1} \cosh \left(\kappa_{1} x\right) \\
A^{(2)}=\alpha_{2} \frac{\cos \left(\kappa_{2} a-x\right)}{\cos \left(\kappa_{2} a\right)}
\end{gathered}
$$

Solutions (30) and (31) must now be sewed at $x=a / 2$ and we have:

$$
A^{(1)}(x=a / 2)=A^{(2)}(x=a / 2) \Rightarrow \alpha_{1} \cosh \left(\kappa_{1} \frac{a}{2}\right)=\alpha_{2} \frac{\cos \left(\kappa_{2} \frac{a}{2}\right)}{\cos \left(\kappa_{2} a\right)}
$$

and

$$
\left.\frac{d A^{(1)}}{d x}\right|_{x=a / 2}=\left.\frac{d A^{(2)}}{d x}\right|_{x=a / 2} \Rightarrow \alpha_{1} \kappa_{1} \sinh \left(\kappa_{1} \frac{a}{2}\right)=\alpha_{2} \kappa_{2} \frac{\sin \left(\kappa_{2} \frac{a}{2}\right)}{\cos \left(\kappa_{2} a\right)}
$$

The ratio of equations (32) and (33) leads to the following dispersion law:

$$
\kappa_{1} \tanh \left(\kappa_{1} \frac{a}{2}\right)=\kappa_{2} \tan \left(\kappa_{2} \frac{a}{2}\right)
$$

For the fundamental mode, for $\kappa_{1} \frac{a}{2}<<1$ and $\kappa_{2} \frac{a}{2}<<1, \tanh \left(\kappa_{1} \frac{a}{2}\right) \approx \kappa_{1} \frac{a}{2}, \tan \left(\kappa_{2} \frac{a}{2}\right) \approx \kappa_{2} \frac{a}{2}$, and we have: $\kappa_{1}^{2}=\kappa_{2}^{2}$

From definitions (27) we get:

$$
\omega^{2}=2 \frac{\pi^{2}}{b^{2}} \frac{c_{1}^{2} c_{2}^{2}}{c_{1}^{2}+c_{2}^{2}}
$$

Equation (35) gives the fundamental frequency of a membrane consisting of two different sections of equal size.

Assuming $c_{1}^{2} \approx c_{2}^{2}$ with $c_{1} \equiv c$ and $c_{2} \equiv c(1-\delta), \delta<<1$, we obtain the following frequency:

$$
\omega=\omega_{1}\left(1-\frac{c_{1}-c_{2}}{2 c_{1}}\right)
$$

If $c_{2}<c_{1}$ (which is the case on the configuration in figure 4), the frequency shifts down, and the absolute value of the relative frequency shift is:

$$
\frac{\omega-\omega_{1}}{\omega_{1}}=-\frac{c_{1}-c_{2}}{2 c_{1}}
$$




\section{$3.2 \quad$ Experiment}

The experimental setup is similar to the previous one in (2.2), with the defect configuration on the specimen as shown in Figure 4. The defect is hand-made in form of crack with a sharp knife, in the width direction; its length is $2 \mathrm{~mm}$. No material is removed. The specimen is preloaded at $14 N$ and has a mean thickness of $h=0.1 \mathrm{~mm}$. The length is $b=550 \mathrm{~mm}$ and the width is $w=12 \mathrm{~mm}$. As an unloaded membrane has no bending stiffness, it is pre-stressed in order to act as a structural element.

The excitation frequency range is 70-500 $\mathrm{Hz}$, with an $8 \mathrm{Vpp}$ sine sweep. The amplitude of the membrane vibration is recorded at the mid-point with the VS-100 OMETRON laser vibrometer.

A frequency analysis with FFT is performed on the received signal. A Hanning window (Von Hann) is applied to reduce leakage and improve amplitude accuracy. The record length is $1 k S$ at a rate of 2 $k S / s$. A leastPrime algorithm is used to display the power spectrum of the signal.

\subsection{Results}

The resonance peak responses for specimen with and without crack are recorded at increasing frequency drive level. An illustration of the frequency response of the material behavior is shown on Figure 5 .

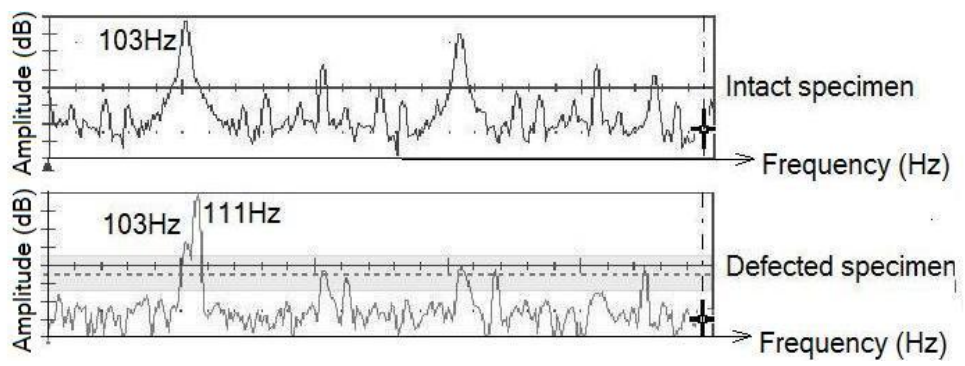

Figure 5: Frequency sweep response. A new peak appears when damage is introduced.

A second spectral line $(111 \mathrm{~Hz})$ appears in the damaged case. It is the scatterer introduced in form of a crack that generates an additional spectral line. This agrees with the prediction in the analytical study. Meanwhile, the new spectral line is unstable in height, exact location and existence, revealing that this new feature (in the spectrum) may be connected to the randomly opening and closing of the defect. Such unstable behaviour of crack was observed recently at vibration of a metallic disk [7]. It was discovered that a crack starts to vibrate in a very narrow range of both amplitude and frequency of the incident wave. After several periods of vibration the corresponding spectral line disappears, and then appears again (see also [8]).

Qualitatively, we therefore observe a duplication of frequency at the fundamental mode as derived in the theoretical part. The table below summarizes the results.

\begin{tabular}{|c|c|c|c|}
\hline & Intact & Damaged (mixing) & Damaged (no mixing) \\
\hline Theory & 118 & 115.7 & 118 and 115.7 \\
\hline Experiment & 103 & 103 and 111 & - \\
\hline
\end{tabular}

Table 2: Fundamental frequencies for intact and damaged specimens with and without frequency mixing. 


\section{$4 \quad$ Finite Element Modeling}

\subsection{Problem description, geometry and model}

Rectangular membranes with and without defect were investigated for natural frequency extraction. The finite element code ABAQUS 6.5 [9] was used to verify the analytical and experimental results. The specimen dimensions and material properties were those given previously.

In the analysis, linear elastic behavior and membrane elements were used to model the material. Membrane elements are used to represent thin surfaces in space that offer strength in the plane of the element but have no bending stiffness. On the other hand, for thin-membrane behavior, the transverse shear and rotary inertia were neglected and an isotropic material behavior was assumed. In this case, only Young's modulus and Poisson's ratio were needed. The element type M3D4R was used to model the material. This is a three-dimensional four-node membrane element with reduced integration, often used for crack meshes problems. However, additional mesh refinement was needed around the defect zone. Reduced integration provides accurate results at a significantly less running time.

The defect in form of a crack was represented as a local deficiency of mass with negligible mass lost compared to the total mass of the material. The corresponding mesh is shown in Figure 6. The defect severity was modeled by moving the nodes at the tips, hence increasing the crack length $a_{0}$, which led to a deformation in connected elements and thus, to a local change in the elements stiffness.

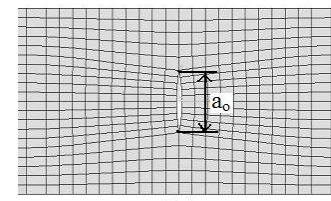

(a)

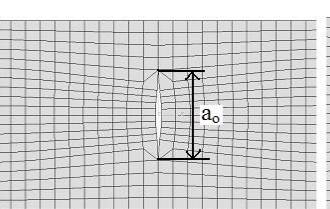

(b)

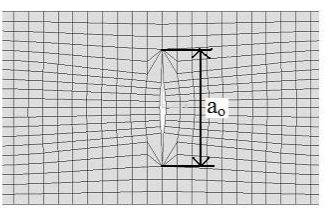

(c)

Figure 6: Mesh around the defect in the FE model. Crack lengths (a): $a_{0}=3.5 \mathrm{~mm}(\mathrm{~b}): a_{0}=4.6 \mathrm{~mm}$ (c): $a_{0}=5.7 \mathrm{~mm}$

The so-called virtual crack extension approach [10] was used in ABAQUS. One of the methods used, the stiffness derivative formulation [11], is a finite element method for inferring energy release rate in bodies. From this formulation, a subsequent work may show that the energy release rate, which is shown to be proportional to the derivative of the stiffness matrix of the material, can be used in this study as a damage indicator.

The defect in form of added mass was modeled as a local increase in thickness on a set of elements covering the defected area, as illustrated in figure 7 .

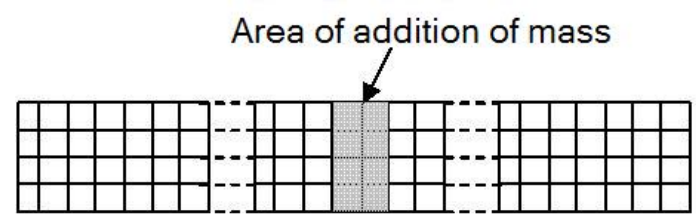

Figure 7: Meshed membrane with an area illustrating an increase in local density of the material.

An eigenvalue analysis was performed with a Frequency step, for natural frequency extraction using the 
Lanczos eigensolver. The boundary condition was defined as specified on the theoretical model.

The dissipation of energy is caused by a number of effects, including friction at the separated faces of the material (crack) and other localized material hysteresis. Damping is a convenient way of including the important absorption of energy without modeling the effects in detail. In ABAQUS/Standard the eigenmodes are calculated for the undamped system, yet most engineering problems involve some kind of damping, however small. This may explain the deviation observed between simulation and experimental or analytical results.

\subsection{Results}

The Figures 8 a-b show that scattering introduction, either in form of added mass or in form of crack, shifts the fundamental frequency, and the relative frequency shift versus percentage of added mass, and normalized crack length has the same trend as observed both in theoretical and experimental parts. The fundamental frequency decreases because, the frequency being inversely proportional to the square root of the mass, locally increasing the mass will shift the fundamental frequency to the left. In case of a crack the frequency shifts to the left as well.
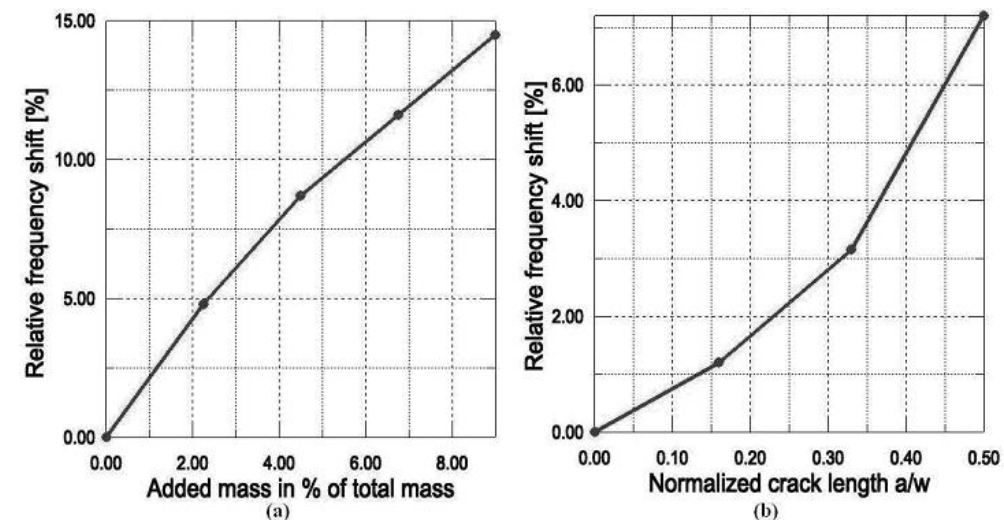

Figure 8: Fundamental frequency shift vs. defect severity for (a):addition of mass; and (b):crack.

\section{Conclusions}

In general, non-destructive testing using vibration-based methods employ natural frequencies, bending modes, resonant frequencies, appearance of new features in the spectral response, and the width of peaks in the frequency spectrum as well as acoustical impedance. These effects are related to mechanical properties such as velocity, stiffness, density or damping, that are important for the integrity of the material. Local changes in mechanical properties that might be related to potential defects cause changes in the measured values and therefore induce shifts of resonance frequencies or broadening of peaks (damping). Results are achieved by comparing the measured values with those of a "healthy" reference material.

This issue is successfully proven in this work. A theoretical model is derived, which qualitatively illustrates the duplication of frequency by a crack, observed from experimental results, for sheet materials. In case of crack, and at low frequency modes, it is difficult to experimentally observe this damage signature, because as stated in previous work [Doebling et. Al.], the natural mode shapes mask the local vibration pattern introduced by the defect. However, a noticeable frequency shift is observed from our 
results, making the approach useful for damage assessment in such materials. A similar observation was recently made on a homogeneous rectangular membrane with different kinds of restraints on different sides [12]. It was observed that a decrease in the natural frequency of a membrane indicates a defect in the membrane fastening. Because of standing wave fields and nodes for a single mode, there is no constant sensitivity over the entire material and defects may not be found sometimes. In that case, the nodes have to be well identified and more than one mode will be measured. In general, the theory developed is convenient for applications such as evaluation of the standard thickness deviations of membranes.

This paper also shows the sensitivity of acoustic weighing for nondestructive testing of sheet materials. The derived theory together with the experiments conducted show how inhomogeneities in form of added mass can be detected using measurement of the frequency shift. Indeed, from equation (16) it is possible to infer that for a membrane vibrating at about $110 \mathrm{~Hz}$, a mass sensitivity $\Delta f / \mathrm{m}^{\prime}$ of the order of $0.25 \mathrm{~Hz} / \mathrm{mg}$ can be considered possible. Meanwhile, the damage may not show up in all but only in some mode shapes. Therefore, the mode-dependent energy dissipation at the defected zone can be revealed by monitoring higher modes, which will lead to the sensitivity of the approach all over the material. The theory developed in this paper is also an accurate tool for that purpose.

\section{Acknowledgement}

The authors are thankful to Prof. I. Yu. Solodov for valuable discussions during the visit of one of the co-authors (E. Mfoumou) at the Institute for Polymer Testing and Polymer Science (IKP) in Stuttgart, Germany. We would also like to give our utmost thanks to Professor O. V. Rudenko from Moscow State University, Russia, for his contribution to this work.

\section{References}

[1] Doherty, J.E., Nondestructive Evaluation, Chapter 12 in Handbook on Experimental Mechanics, A. S. Edt, Society for Experimental Mechanics, inc. 1988.

[2] Doebling, S.W., Farrar, C.R., and Prime, M.B., Summary Review of Vibration-Based Damage Identification Methods, Shock and Vibration Digest, 30 (2), 91-105. 1998.

[3] Hagemaier, D.J. and Fassbender, R.H, Nondestructive testing of advanced composites, Materials Evaluation 37 pp 43-49(1979).

[4] Landau L.D., and Lifshitz E.M., Theory of Elasticity, 3rd Edition, Pergamon, 1979.

[5] Sauerbrey, G., Use of Vibrating Quartz for Thin Film Weighting and Microweighting, (in German), Z. Phys., 155, 206-222, 1959.

[6] King, W. H., Piezoelectric Sorption Detector, Anal. Chem., 36 (9), 1735-1739, 1964.

[7] Korobov, A. I., Izosimova, M. Yu, Nonlinear Lamb Waves in a Metal Plate with Defects, Acoustical Physics, Vol. 52, No. 5, pp 589-597, 2006.

[8] Rudenko, O. V., Giant Nonlinearities of Structurally-inhomogeneous Media and Principles of Nonlinear Acoustic Diagnostics (a review), Physics-Uspekhi (Advances in Physical Sciences), Vol. 176, No.1, pp. 77-95, 2006. 
[9] ABAQUS/Standard User's Manual. Volume I-III Version 6.5, Hibbit, Karlsson and Sorensen, Inc., 2004.

[10] Hellen, T.K., On the Method of Virtual Crack Extensions, International Journal for Numerical Methods in Engineering, Vol. 9, pp. 187-207, 1975.

[11] Parks, D.M., A Stiffness Derivative Finite Element Technique for Determination of Crack Tip Stress Intensity Factors, International Journal of Fracture, Vol. 10, pp. 487-502, 1974.

[12] Akhtyamov, A. M., Identification of the Boundary Conditions of a Rectangular Membrane from its Natural Frequencies, Acoustical Physics, Vol. 52, No. 3, 2006. 